\title{
Twistor spaces of algebraic dimension two associated to a connected sum of projective planes
}

\author{
Akira Fujiki
}

\begin{abstract}
For any integer $m \geqslant 4$ we construct twistor spaces of algebraic dimension $a=2$ associated to some self-dual structures on $m \boldsymbol{P}^{2}$, the connected sum of $m$ complex projective planes $\boldsymbol{P}^{2}$. Together with previously known results this implies that for any $m \geqslant 5$ all the possible values for algebraic dimension $0 \leqslant a \leqslant 3$ are attained for some such twistor spaces. Our method is to construct such twistor spaces by small deformations of Joyce twistor spaces.
\end{abstract}

\section{Introduction}

Let $m$ be a positive integer and $M:=m \boldsymbol{P}^{2}$ the smooth connected sum of $m$ copies of the complex projective plane $\boldsymbol{P}^{2}$. Suppose that we are given a self-dual conformal class $[g]$ on $M$. Assume that $[g]$ is of positive type in the sense that the conformal class $[g]$ contains a Riemannian metric whose scalar curvature is a positive constant. Let $Z$ be the twistor space associated to this self-dual manifold $(M,[g])$. Then $Z$ is a three-dimensional compact complex manifold which fibers smoothly over $M$ with fiber isomorphic to the complex projective line $\boldsymbol{P}$. We are interested in the algebraic dimension $a(Z)$ of $Z$, which is by definition the transcendence degree of the field of meromorphic functions on $Z$, and which can take one of the values $0,1,2$ or 3 . As for the problem as to which of these values can actually be attained for some twistor spaces as above, the following results are known:

1) We have $a(Z)=3$ if $m \leqslant 3$, and $a(Z) \geqslant 1$ if $m=4$.

2) For each $m \geqslant 1$ LeBrun [LeB91] and Joyce [Joy95] have explicitly constructed families of self-dual metrics on $m \boldsymbol{P}^{2}$ whose twistor spaces turn out to be Moishezon, that is, $a(Z)=3$ (cf. [LeB91, Joy95, Fuj00]).

3) For each $m \geqslant 5$ there exist twistor spaces $Z$ with $a(Z)=0$ (see [DF89, LP92, Cam91]), and similarly for each $m \geqslant 4$ there exist twistor spaces $Z$ with $a(Z)=1$ (see [LP92, Cam94, Poo92, Hon99]).

4) For $m=4$ there exist twistor spaces $Z$ with $a(Z)=2$ (see [CK99]).

A natural problem thus arises as to whether there exists a twistor space $Z$ with $a(Z)=2$ in the case $m \geqslant 5$. The purpose of this paper is to show that the answer to this problem is affirmative. Namely we prove the following Main Theorem.

Main Theorem. For any $m \geqslant 4$ there exist twistor spaces $Z$ associated to a self-dual conformal class of positive type on $m \boldsymbol{P}^{2}$ with $a(Z)=2$.

Received 25 October 2002, accepted in final form 20 June 2003.

2000 Mathematics Subject Classification 32J17 (primary), 32J25, 53C15 (secondary).

Keywords: twistor space, self-dual manifold, algebraic dimension.

The author is supported by a Grant-in-Aid for Scientific Research (No. 12440019).

This journal is (C) Foundation Compositio Mathematica 2004. 


\section{A. FujIKI}

The examples in results 3 and 4 are all obtained as small deformations of a 'general' LeBrun twistor space in [LeB91] and the main line of arguments here is basically the same, except that we use small deformations of Joyce twistor spaces studied in detail in [Fuj00], which includes as a special case the 'degenerate' case of the LeBrun twistor spaces. We note that if $a(Z)=2$ for a twistor space $Z$ of a compact self-dual manifold $(M,[g])$ in general, then $M$ is homeomorphic either to $m \boldsymbol{P}^{2}$ or to $\left(S^{1} \times S^{3}\right) \# m \boldsymbol{P}^{2}$ and $[g]$ is of positive type (cf. [Fuj02] for more details).

After some preliminaries concerning symmetric toric surfaces in $\S 2$, we construct in $\S 3$ some projective nonsingular rational surfaces with anti-Kodaira dimension one as a local deformation of a symmetric toric surface. In $\S 4$ we basically show that a twistor space is of algebraic dimension two if it contains as a fundamental divisor one such rational surface. In $\S 5$ we prove a general unobstructedness result for local deformations of a pair consisting of a twistor space and a smooth fundamental divisor on it. Finally in $\S 6$ we consider a pair of a Joyce twistor space and a smooth fundamental divisor on it which is a symmetric toric surface. We apply the result of the previous section to such a pair and show that a certain deformation of the pair gives rise to a twistor space which contains one of the rational surfaces in $\S 3$ as a fundamental divisor. The Main Theorem then follows.

Notations. For a line bundle $L$ on a complex manifold $X$ we denote by $|L|$ the corresponding linear system of divisors on $X$. For a vector bundle $E$ on $X$ we write $h^{i}(E)=h^{i}(X, E)=\operatorname{dim} H^{i}(X, E)$ and $\chi(X, E)=\sum_{i}(-1)^{i} h^{i}(E)$.

\section{Preliminaries}

2.1 Let $B$ be a cycle of nonsingular rational curves, i.e. $B$ is a compact curve whose irreducible components $B_{1}, \ldots, B_{l}$ are nonsingular rational curves such that $B_{i}$ and $B_{i+1}$ intersect transversally at a single point $p_{i}$ for $1 \leqslant i \leqslant l$, where $B_{l+1}=B_{1}$, and that there exist no other intersections among the irreducible components of $B$. The natural map $H^{1}\left(B, C^{*}\right) \rightarrow H^{1}\left(B, O_{C}^{*}\right)$ induces an isomorphism $H^{1}\left(B, C^{*}\right) \cong \mathrm{Pic}_{0} B$, the identity component of the Picard group Pic $B$ of $B$. Moreover, we have $H^{1}\left(B, C^{*}\right) \cong C^{*}$.

We fix a generator $g$ of the fundamental group $\pi_{1} \cong \boldsymbol{Z}$ of $B$. Suppose that there exists an anti-holomorphic involution $\sigma$ of $B$. It induces an involution $\sigma_{*}$ of $\pi_{1}$ and we have $\sigma_{*}(g)=g^{\varepsilon}$, where $\varepsilon=1$ or -1 . Also $\sigma$ induces an anti-holomorphic involution of Pic $B$ which is an automorphism of a group. Denote by $\operatorname{Pic}^{R} B$ the fixed subgroup of this action and set $\operatorname{Pic}_{0}^{R} B=\operatorname{Pic}^{R} B \cap \operatorname{Pic}_{0} B$. A line bundle $L$ on $B$ defines a point of $\operatorname{Pic}^{R} B$ if and only if $\sigma$ lifts to an anti-holomorphic bundle automorphism $\tilde{\sigma}$ of $L$. (A priori $\tilde{\sigma}$ may not be an involution.)

Lemma 2.1. Suppose that $\varepsilon=1$ (respectively -1). Then $\operatorname{Pic}_{0}^{R} B \cong \boldsymbol{R}^{*}$ (respectively $S^{1}$ ) with respect to a natural isomorphism $\operatorname{Pic}_{0} B \cong C^{*}$. Moreover, for any line bundle $L$ in $\operatorname{Pic}_{0}^{R} B$ the lift $\tilde{\sigma}$ as above can be taken to be an involution.

Proof. First note that an anti-holomorphic involution of $\boldsymbol{C}^{*}$ as a group is either $t \rightarrow \bar{t}$ or $t \rightarrow \bar{t}^{-1}$, where the bar denotes the complex conjugation; in the former (respectively latter) case the real part is isomorphic to $\boldsymbol{R}^{*}$ (respectively $S^{1}$ ). Now let $p: \tilde{B} \rightarrow B$ be the universal covering map, whose covering transformation group is identified with $\pi_{1}$. We fix a lift $\tilde{\sigma}$ of $\sigma$ to an anti-holomorphic automorphism of $\tilde{B}$. Then we have the equality $\tilde{\sigma} g \tilde{\sigma}^{-1}=g^{\varepsilon}$ of biholomorphic automorphisms of $\tilde{B}$. For any element $b \in C^{*}$ we define the action of $\pi_{1}$ on the product $\tilde{B} \times \boldsymbol{C}$ by $\hat{g}((\tilde{x}, \zeta))=(g(\tilde{x}), b \zeta)$, $\tilde{x} \in \tilde{B}, \zeta \in \boldsymbol{C}$. Then the quotient $L_{b}:=(\tilde{B} \times \boldsymbol{C}) / \pi_{1}$ is a flat line bundle on $B$ and the map $\boldsymbol{C}^{*} \rightarrow$ Pic $_{0} B, b \rightarrow L_{b}$, gives a group isomorphism. Let the anti-holomorphic automorphism $\tilde{\sigma}$ be lifted to an anti-holomorphic bundle automorphism $\hat{\sigma}$ of $\tilde{B} \times C$ via $\hat{\sigma}(\tilde{x}, \zeta)=(\tilde{\sigma}(\tilde{x}), \bar{\zeta})$. This automorphism then descends to one of $L_{b}$ on $B$ if the equality $\hat{\sigma} \hat{g} \hat{\sigma}^{-1}=\hat{g}^{\varepsilon}$ for $\hat{g}=\hat{g}_{b}$ holds, which is equivalent to 


\section{TWistor SPACES OF ALGEBRAIC DIMENSION TWO}

the condition $b=\bar{b}$ (respectively $b=\bar{b}^{-1}$ ). Together with the remark at the beginning of the proof, this implies the first assertion of the lemma. The second assertion is clear from the above proof.

Suppose now that $l=2 m$ is even and that for any $1 \leqslant i \leqslant m$ the anti-holomorphic involution $\sigma$ of $B$ interchanges $B_{i}$ and $B_{i+m}$. Suppose further that $B$ is embedded in a smooth complex surface $T$ and $\sigma$ is induced by an anti-holomorphic involution on $T$ (still denoted by $\sigma$ ). Let $\operatorname{Pic}_{c} B$ be the connected component of Pic $B$ consisting of the line bundles which have the same Chern class with $K_{T}^{-1} \mid B$, where $K_{T}$ is the canonical bundle of $T$. Set $\operatorname{Pic}_{c}^{R} B=\operatorname{Pic}^{R} B \cap \operatorname{Pic}_{c} B$.

LEMma 2.2. Let the notations and assumptions be as above. Then there exists a natural isomorphism $\operatorname{Pic}_{c} B \cong C^{*}$ inducing $\operatorname{Pic}_{c}^{R} B \cong \boldsymbol{R}^{*}$. Moreover, for any line bundle $F$ in $\operatorname{Pic}_{c}^{R} B, \sigma$ induces a natural anti-holomorphic involution on the projective space $\boldsymbol{P} H^{0}(B, F):=\left(H^{0}(B, F)-0\right) / \boldsymbol{C}^{*}$ which is induced by a complex conjugation on the vector space $H^{0}(B, F)$.

Proof. Under our assumption on $\sigma$ we see immediately that $\sigma$ induces the identity of $\pi_{1}$ and hence $\operatorname{Pic}_{0}^{R} B \cong \boldsymbol{R}^{*}$ by Lemma 2.1. The natural isomorphism $v: \operatorname{Pic}_{0} B \rightarrow \operatorname{Pic}_{c} B$ induced by the multiplication by $K_{T}^{-1} \mid B$ is $\sigma$-equivariant since $K_{T}^{-1}$ is $\sigma$-invariant. Indeed $\sigma$ on $T$ lifts naturally to an anti-holomorphic involution on $K_{T}^{-1}$. The first assertion follows. Moreover, combined with the second assertion of Lemma 2.1 it follows that any $F$ as in the lemma admits a lift $\tilde{\sigma}$ of $\sigma$ which is an involution, $F$ being a product of $K_{T}^{-1} \mid B$ and an element of $\operatorname{Pic}_{0}^{R} B$. From this the second assertion follows.

2.2 Let $S$ be a projective nonsingular rational surface and $C=\bigcup_{1 \leqslant j \leqslant 2 k} C_{j}$ a cycle of nonsingular rational curves embedded in $S$. (The numbering is in such a way that $C_{i} \cap C_{i+1}=\left\{p_{i}\right\}, 1 \leqslant i \leqslant 2 k$, and that there exist no other intersections, where $C_{2 k+1}=C_{1}$.) Suppose that $S$ admits a fixedpoint-free anti-holomorphic involution $\sigma$ which interchanges $C_{i}$ and $C_{i+k}$ for all $i$ with $1 \leqslant i \leqslant k$. We call such a $\sigma$ a real structure of $S$, or more precisely of $(S, C)$. If $u: S^{\prime} \rightarrow S$ is the blowing-up of $S$ with center $p_{i}$ and $p_{i+k}$ for some $i$, and $C^{\prime}=u^{-1}(C)$ with reduced structure, $\sigma$ lifts to a real structure of $\left(S^{\prime}, C^{\prime}\right)$ in the sense defined above. We call such a blowing-up admissible and call admissible any bimeromorphic morphism which is a finite succession of admissible blowing-ups.

By a toric surface we shall mean here a projective nonsingular rational surface $S$ on which the algebraic torus $C^{*} \times C^{*}$ acts algebraically with an open orbit. The complement $C$ of the open orbit $U$ consists of a cycle of nonsingular rational curves and is a member of the anti-canonical system $\left|K_{S}^{-1}\right|$. The complement $C$ is called the anti-canonical cycle of $S$. If $(S, C)$ admits a real structure, we have $C_{i}^{2}=C_{i+k}^{2}$ for all $i, 1 \leqslant i \leqslant k$, that is, $S$ is a symmetric toric surface in the sense of [Fuj00, (2.5)].

Let $\boldsymbol{P}=\boldsymbol{C}(z) \cup\{\infty\}$ be the complex projective line. Let $S_{0}:=\boldsymbol{P} \times \boldsymbol{P}$ and $p_{i}: S_{0} \rightarrow \boldsymbol{P}$ be the $i$ th projections, $i=1,2$. Set $\bar{C}_{1}=p_{1}^{-1}(0), \bar{C}_{2}=p_{2}^{-1}(0), \bar{C}_{3}=p_{1}^{-1}(\infty)$, and $\bar{C}_{4}=p_{2}^{-1}(\infty)$. On $\boldsymbol{P}$ we have anti-holomorphic involutions $\sigma_{1}: z \rightarrow 1 / \bar{z}$ and $\sigma_{2}: z \rightarrow-1 / \bar{z}$. We consider $S_{0}$ naturally as a toric surface with open orbit $S_{0}-\bar{C}$ and with real structure $\sigma$ on $\left(S_{0}, \bar{C}\right)$ defined by $\sigma_{1} \times \sigma_{2}$, where $\bar{C}=\bigcup_{j=1}^{4} \bar{C}_{j}$. If $f: S \rightarrow S_{0}$ is any admissible bimeromorphic morphism, $S$ is again a toric surface with naturally induced real structure.

\section{Rational surfaces with anti-Kodaira dimension one}

Let $S$ be a projective nonsingular surface and set $A=\left\{m \geqslant 0:\left|K_{S}^{-m}\right| \neq \emptyset\right\}$. When $A$ is nonempty, the anti-Kodaira dimension $\kappa^{-1}(S)$ of $S$ is defined to be

$$
\kappa^{-1}(S):=\max _{m \in A} \operatorname{dim} f_{m}(S),
$$

where $f_{m}$ is the rational map defined by the anti-canonical system $\left|K_{S}^{-m}\right|$ and $f_{m}(S)$ denotes the rational image of $S$ by $f_{m}$. If $A$ is empty, we set $\kappa^{-1}(S)=-\infty$. The purpose of this section is to 


\section{A. FuJiki}

construct a family of rational surfaces with anti-Kodaira dimension one by deforming certain real symmetric toric surfaces.

Suppose that $S$ is a projective nonsingular rational surface with $\left|K_{S}^{-1}\right| \neq \emptyset$. Let $C$ be a connected curve in $\left|K_{S}^{-1}\right|$. Denote by $N:=[C] \mid C$ the normal bundle of $C$ in $S$ so that $N \cong K_{S}^{-1} \mid C$. We assume that $N$ is a torsion line bundle on $C$ of order $n \geqslant 1$.

Lemma 3.1. Let the notations and assumptions be as above. Then we have

$$
h^{0}\left(K_{S}^{-l}\right)=1, \quad 1 \leqslant l<n, \quad \text { and } \quad h^{0}\left(K_{S}^{-n}\right)=2 .
$$

$\left|K_{S}^{-n}\right|$ induces a structure $g: S \rightarrow \boldsymbol{P}$ of an elliptic surface on $S$. When $n>1, C$ is the unique member of $\left|K_{S}^{-1}\right|$ and it is the support of a unique multiple fiber of $g$, whose multiplicity is $n$.

Proof. By using the Riemann-Roch theorem we have

$$
H^{0}\left(C, N^{k}\right)=H^{1}\left(C, N^{k}\right)=0 \quad \text { for } 1 \leqslant k \leqslant n-1 .
$$

Let $I$ be the ideal sheaf of $C$ in $S$. For any integer $l>0$ we denote by $C_{(l)}$ the subspace of $S$ defined by the ideal sheaf $I^{l}$. Then we have the short exact sequence

$$
\left.0 \rightarrow O_{S} \rightarrow K_{S}^{-l} \rightarrow K_{S}^{-l}\right|_{C_{(l)}} \rightarrow 0,
$$

and the associated long exact sequence

$$
0 \rightarrow H^{0}\left(S, O_{S}\right) \rightarrow H^{0}\left(S, K_{S}^{-l}\right) \rightarrow H^{0}\left(C_{(l)}, K_{S}^{-l} \mid C_{(l)}\right) \rightarrow 0 .
$$

We compute $H^{0}\left(C_{(l)}, K_{S}^{-l} \mid C_{(l)}\right)$ for $l \leqslant n$. Noting that $N^{l-k} \cong I^{k} / I^{k+1} \otimes K_{S}^{-l}$ we have

$$
0 \rightarrow N^{l-k} \rightarrow K_{S}^{-l}\left|C_{(k+1)} \rightarrow K_{S}^{-l}\right| C_{(k)} \rightarrow 0 .
$$

Using (2) we then get

$$
H^{0}\left(C, K_{S}^{-l} \mid C\right) \cong \cdots \cong H^{0}\left(C_{(l)}, K_{S}^{-l} \mid C_{(l)}\right) \text { for any } l \leqslant n,
$$

while $h^{0}\left(C, K_{S}^{-l} \mid C\right)=1$ if $l=n$, and $=0$ otherwise. Thus by (3) we have $h^{0}\left(S, K_{S}^{-n}\right)=2$ and $h^{0}\left(S, K_{S}^{-l}\right)=1$ if $1 \leqslant l<n$. Therefore we get the first assertion. Also, (3) and (4) for $l=n$ show that $\left|K_{S}^{-n}\right|$ is base-point-free and $C_{(n)}$ is a fiber of the associated morphism $g: S \rightarrow \boldsymbol{P}$ which induces an elliptic surface structure on $S$. When $n>1, C_{(n)}$ is a multiple fiber with multiplicity $n$. Finally, on a rational surface there exists at most one multiple fiber (cf. [Dol81, p. 133, Corollary 2]).

Consider the toric surface $S_{0}:=\boldsymbol{P} \times \boldsymbol{P}$ with its real structure $\sigma$ given as in $\S 2.2$. Denote by $\alpha: T \rightarrow S_{0}$ the blowing-up of the four nodes of $\bar{C}$, and by $C_{i}, 1 \leqslant i \leqslant 4$, the proper transforms of $\bar{C}_{i}$ in $T$. We further define $E_{i}$ to be the inverse images of the nodes $\bar{C}_{i} \cap \bar{C}_{i+1}$, where $\bar{C}_{5}=\bar{C}_{1}$. Then we set $q_{i}=E_{i} \cap C_{i}$, and $r_{i}=E_{i} \cap C_{i+1}$. The real structure $\sigma$ on $S_{0}$ lifts to one on $T$, which is again denoted by $\sigma$. For $i=1,2, \sigma$ interchanges members of each of the pairs $\left(C_{i}, C_{i+2}\right),\left(E_{i}, E_{i+2}\right)$, $\left(q_{i}, q_{i+2}\right)$ and $\left(r_{i}, r_{i+2}\right)$ respectively. Let $B:=\alpha^{-1}(\bar{C})$ with reduced structure, which is the union of $C_{i}$ and $E_{j}, 1 \leqslant i, j \leqslant 4$. Then $T$ is again a toric surface with anti-canonical cycle $B$.

Now we take an admissible bimeromorphic morphism $u: T^{\prime} \rightarrow T$, where we include the case where $T^{\prime}=T$ and $u$ is the identity. Again $T^{\prime}$ is a toric surface with anti-canonical cycle $B^{\prime}$ given by the reduced inverse image of $B$ and with induced real structure (still denoted by $\sigma$ ).

Let $E_{i}^{\prime}$ be the proper transform of $E_{i}$ in $T^{\prime}$. Then $u$ induces an isomorphism $E_{i}^{\prime} \cong E_{i}$. In general we denote a point of $E_{i}^{\prime}$ corresponding to a point $p$ of $E_{i}$ by $p^{\prime}$; for example, we may speak of the points $q_{i}^{\prime}$ and $r_{i}^{\prime}$ of $E_{i}^{\prime}$. We consider the product

$$
E:=E_{1} \times E_{2} \times E_{3} \times E_{4}
$$




\section{TWistor SPACES OF ALGEBRAIC DIMENSION TWO}

and the holomorphic family $\left\{S_{a}\right\}_{a \in E}$ of nonsingular rational surfaces $S_{a}$ parametrized by $a=$ $\left(a_{1}, a_{2}, a_{3}, a_{4}\right) \in E, a_{i} \in E_{i}$, where $S_{a}$ is obtained from $T^{\prime}$ by blowing up the four points $a_{1}^{\prime}, a_{2}^{\prime}, a_{3}^{\prime}$ and $a_{4}^{\prime}$ of $T^{\prime}$. In general $S_{a}$ is not a toric surface any more.

Suppose that $a_{i} \neq q_{i}, r_{i}$ for any $i$. Then we may also view the surface $S_{a}$ as follows. Let $v_{a}$ : $T_{a} \rightarrow T$ be the blowing-up of $a_{i}, 1 \leqslant i \leqslant 4$. Since $a_{i} \neq q_{i}, r_{i}$, there exists an admissible birational morphism $u_{a}: S_{a} \rightarrow T_{a}$ such that $v_{a} u_{a}=u w_{a}$, i.e.

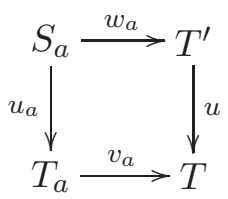

where $w_{a}: S_{a} \rightarrow T^{\prime}$ is the natural map.

Now we show the following lemma.

Lemma 3.2. Suppose that $a_{i} \neq q_{i}, r_{i}$ for any $i$ as above. Let $F_{a}$ be the line bundle on $B$ defined by the Cartier divisor $a_{1}+a_{2}+a_{3}+a_{4}$ on $B$. Suppose further that

$$
L_{a}:=F_{a}^{-1} \otimes\left(K_{T}^{-1} \mid B\right) \in \operatorname{Pic}_{c} B
$$

is a torsion line bundle on $B$. Then we have $h^{0}\left(K_{S_{a}}^{-1}\right) \geqslant 1$ and $\kappa^{-1}\left(S_{a}\right)=1$. The case $h^{0}\left(K_{S_{a}}^{-1}\right)>1$ occurs if and only if $u: T^{\prime} \rightarrow T$ is the identity and $L_{a}$ is trivial. Moreover, in this case $h^{0}\left(K_{S_{a}}^{-1}\right)=2$.

Proof. We first study the structure of $T_{a}$. Let $n$ be the order of $L_{a}$ on $B$. Let $B_{a}$ be the proper transform of $B$ in $T_{a}$. Then since $a_{i} \neq q_{i}, r_{i}, v_{a} \mid B_{a}: B_{a} \rightarrow B$ is isomorphic, and $B_{a}$ is again an anti-canonical cycle of $T_{a}$. In particular as a line bundle we have

$$
K_{T_{a}}^{-1}\left|B_{a} \cong\left[B_{a}\right]\right| B_{a}
$$

where $K_{T_{a}}$ is the canonical bundle of $T_{a}$. On the other hand, if we denote the exceptional curve of $v_{a}$ by $D_{a}:=\bigcup_{1 \leqslant i \leqslant 4} v_{a}^{-1}\left(a_{i}\right)$ we have $\left[D_{a}\right] \mid B_{a} \cong\left(v_{a} \mid B_{a}\right)^{*} F_{a}$. Therefore we get

$$
K_{T_{a}}^{-1}\left|B_{a} \cong\left(v_{a}^{*} K_{T}^{-1} \otimes\left[D_{a}\right]^{-1}\right)\right| B_{a} \cong\left(v_{a} \mid B_{a}\right)^{*}\left(K_{T}^{-1} \mid B \otimes F_{a}^{-1}\right)=\left(v_{a} \mid B_{a}\right)^{*} L_{a}
$$

By (7), (8) and our assumption, $\left[B_{a}\right] \mid B_{a}$ is thus a torsion line bundle of order $n$. Then by Lemma 3.1, $\left|K_{T_{a}}^{-n}\right|$ defines a holomorphic map $f_{a}: T_{a} \rightarrow \boldsymbol{P}$ making $T_{a}$ an elliptic surface over $\boldsymbol{P}$ such that $B_{a(n)}$ is the unique multiple fiber of $f_{a}$.

Using this we next show that $\kappa^{-1}\left(S_{a}\right)=1$. Since the morphism $u_{a}: S_{a} \rightarrow T_{a}$ is admissible, the inverse image $C_{a}:=u_{a}^{-1}\left(B_{a}\right)$ of $B_{a}$ in $S_{a}$ with reduced structure is a member of $\left|K_{S_{a}}^{-1}\right|$ and $C_{a}$ is the support of a fiber of the induced elliptic surface structure $f_{a} u_{a}: S_{a} \rightarrow \boldsymbol{P}$. In particular $h^{0}\left(K_{S_{a}}^{-1}\right) \geqslant 1$, and we have the equalities

$$
\kappa^{-1}\left(S_{a}\right)=\kappa\left(\left[C_{a}\right] ; S_{a}\right)=\kappa\left([Q] ; S_{a}\right)=1,
$$

where $\kappa\left(L ; S_{a}\right)$ denotes the Iitaka dimension of a line bundle $L$ on $S_{a}$ in general [Uen75] and $Q$ denotes a general fiber of $f_{a} u_{a}$.

Finally, when $u_{a}$ is not isomorphic, the member $C_{a}$ of $\left|K_{S_{a}}^{-1}\right|$ is reduced and is a support of a fiber, but does not coincide with the fiber. It follows that $C_{a}$ cannot move and hence $\operatorname{dim} h^{0}\left(K_{S_{a}}^{-1}\right)=1$. Suppose next that $u_{a}$ is isomorphic so that $S_{a} \cong T_{a}$ and $T^{\prime} \cong T$. In this case if $\operatorname{dim}\left|K_{T_{a}}^{-1}\right|>0$ and $B_{a}^{\prime}$ is any member other than $B_{a}$, we must have $B_{a} \cap B_{a}^{\prime}=0$ since $K_{T_{a}}^{-1} \mid B_{a}$ is torsion. This implies that $K_{T_{a}}^{-1} \mid B_{a}$ is trivial, or $L_{a}$ is trivial on $B$ by (8). Conversely, if this condition is satisfied, we have $h^{0}\left(K_{T_{a}}^{-1} \mid B_{a}\right)=1$, so that by the sequence (3) applied to $S=T_{a}$ and $l=1$ we have $h^{0}\left(K_{T_{a}}^{-1}\right)=2$.

Let $U \cong C^{* 4}$ be the Zariski open subset of $E$ consisting of those points $a=\left(a_{1}, a_{2}, a_{3}, a_{4}\right)$ for which $a_{i} \neq q_{i}, r_{i}$ for any $i$. Let $\operatorname{Pic}_{c} B$ be the connected component of Pic $B$ containing $K_{T}^{-1} \mid B$ as 


\section{A. FuJiki}

defined in $\S 2.1$. The real structure $\sigma$ of $T$ induces anti-holomorphic involutions of $E, U, \mathrm{Pic}_{c} B$, etc., all denoted still by $\sigma$ and referred to as real structures. Note that the induced real structure on $E$ interchanges $E_{1}$ and $E_{3}$, and $E_{2}$ and $E_{4}$. A point which is fixed by $\sigma$ is called a real point. Pic $_{c}^{R} B$ is thus the set of real points on $\mathrm{Pic}_{c} B$. Now we consider the special real point $a^{\#}:=\left(q_{1}, r_{2}, q_{3}, r_{4}\right)$ of $E$.

Lemma 3.3. For any line bundle $F$ in $\operatorname{Pic}_{c} B$ and for any neighborhood $V$ of $a^{\#}$ in $E$ there exists a point $a=\left(a_{1}, a_{2}, a_{3}, a_{4}\right)$ of $U \cap V$ such that the associated line bundle $F_{a}$ on $B$ is isomorphic to $F$. If $F$ belongs to $\operatorname{Pic}_{c}^{R} B$, we may take $a$ to be a real point.

Proof. For any element $F \in \operatorname{Pic}_{c} B$ we have $\operatorname{deg} F \mid E_{i}=1$ and $\operatorname{deg} F \mid C_{j}=0$ for any $i$ and $j$. By Serre duality and by our assumption we have $h^{1}(B, F)=h^{0}\left(B, F^{-1}\right)=0$ in view of the triviality of $K_{B}:=\left(K_{T} \otimes[B]\right) \mid B$. Then by the Riemann-Roch theorem we get $h^{0}(B, F)=4$. In fact it is readily shown that the restriction map $H^{0}(B, F) \rightarrow \bigoplus_{j} H^{0}\left(C_{j}, F \mid C_{j}\right) \cong C^{4}$ is isomorphic. Let $p_{j}: H^{0}(B, F) \rightarrow H^{0}\left(C_{j}, F \mid C_{j}\right) \cong C$ be the induced projection, and $K_{j}(F)$ its kernel. Then we obtain a $\boldsymbol{P}^{3}$-bundle $h: W \rightarrow \operatorname{Pic}_{c} B$ whose fiber over $F \in \mathrm{Pic}_{c} B$ is the projectified vector space $\boldsymbol{P} H^{0}(B, F):=\left(H^{0}(B, F)-\{0\}\right) / \boldsymbol{C}^{*}$. Moreover we obtain its $\boldsymbol{P}^{2}$-subbundles $W_{j} \rightarrow \mathrm{Pic}_{c} B, 1 \leqslant$ $j \leqslant 4$, with fibers $\boldsymbol{P} K_{j}(F) \subseteq \boldsymbol{P} H^{0}(B, F)$.

Let $U_{0}:=W-\bigcup_{j} W_{j}$. Note that a point of $W$ belongs to $\bigcup_{j} W_{j}$ if and only if a representative of it in $H^{0}(B, F)$ vanishes at least one of the points in $a^{\#}$; in fact since $\operatorname{deg} F \mid C_{j}=0$, if a section of $F$ vanishes at a point of $C_{j}$ it vanishes identically on $C_{j}$. Then we have a natural biholomorphic map $g: U_{0} \rightarrow U$ of $U_{0}$ onto $U$, where a point of $U_{0}$ represented by a section $s$ of $F$, which never vanishes on any of the points in $a^{\#}$ by the above remark, corresponds to the point $\left(a_{1}, a_{2}, a_{3}, a_{4}\right)$ of $U, a_{i}$ being the unique zero of $s$ on $E_{i}-\left\{q_{i}, r_{i}\right\}$.

Now for any neighborhood $V$ of $a^{\#}$ in $E$ if we take a sufficiently small neighborhood $N$ of $W_{1} \cap W_{3}$ in $W$ we have $g\left(N \cap U_{0}\right) \subseteq V$ since points of $W_{1} \cap W_{3}$ are represented by sections which vanish at all points in $a^{\#}$. Any point $a$ of the set $g\left(N \cap U_{0} \cap h^{-1}(F)\right)$, which is clearly nonempty, then meets the requirement of the lemma.

Suppose finally that $F$ belongs to $\operatorname{Pic}_{c}^{R} B$. Then $E$ and $W$ have natural real structures of which $U$ and $U_{0}$ are $\sigma$-invariant open subsets respectively. Moreover, $g$ and $h$ are compatible with real structures. By Lemma 2.2 the real part $h^{-1}(F)_{R}$ of $h^{-1}(F)$ is diffeomorphic to a three-dimensional real projective space and it has nonempty intersection with the $\sigma$-invariant $\boldsymbol{P}$-bundle $W_{1} \cap W_{3}$ over $\operatorname{Pic}_{c} B$. Thus $N \cap h^{-1}(F)_{R}$ is never empty, and we can take any point $a$ of $g\left(N \cap h^{-1}(F)_{R}\right)$ as a desired real point.

Corollary 3.4. Let $n$ be a positive integer. Then for any neighborhood $V$ of $a^{\#}$ in $E$ there exists a point a of $U \cap V$ such that the corresponding line bundle $L_{a}(c f .(6))$ is a torsion line bundle on $B$ of order $n$. If $n=1$ or 2 , we can take $a$ to be a real point in $U \cap V$.

Proof. For the first assertion we have only to take $F$ in the lemma in such a way that $F^{-1} \otimes K_{T}^{-1} \mid B$ is a torsion line bundle of order $n$ on $B$ and then apply the above lemma to $F$. When $n=1$ or $2, F^{-1} \otimes K_{T}^{-1} \mid B$, and hence $F$ also, is real and the assertion again follows from the above lemma.

For later reference we shall summarize what we have obtained in this section in terms of deformation of surfaces. Let $S$ be the toric surface with real structure $\sigma$ obtained from $T^{\prime}$ by blowing up the four points $q_{1}^{\prime}, r_{2}^{\prime}, q_{3}^{\prime}$ and $r_{4}^{\prime}$, i.e. $S=S_{a^{\#}}$ for $a^{\#}=\left(q_{1}, r_{2}, q_{3}, r_{4}\right)$. For a neighborhood $V$ of $a^{\#}$ in $E$ we consider the surfaces $S_{a}, a \in V$, as small deformations of $S$. The surface $S_{a}$ contains a cycle $C_{a}$ of nonsingular rational curves and the point $a$ defines a holomorphic line bundle $L_{a}$ on the cycle of nonsingular rational curves $B$ on $T$ by the formula (6). 


\section{TWistor SPACES OF ALGEBRAIC DIMENSION TWO}

Proposition 3.5. Let $n$ be any positive integer. Then for any neighborhood $V$ of $a^{\#}$ as above, we can find a point $a \in U \cap V$ such that $L_{a}$ is a torsion line bundle of order $n$ and $\kappa^{-1}\left(S_{a}\right)=1$. Moreover, if $n=1$ or 2 , we can take $S_{a}$ to be a real surface with real structure $\sigma_{a}$ so that $\left(S_{a}, \sigma_{a}\right)$ is a deformation of $(S, \sigma)$ with real smooth parameters.

Note that the last statement follows from the proof of Lemma 3.3.

\section{Algebraic dimension of $Z$ and anti-Kodaira dimension of $S$}

Let $Z$ be the twistor space associated to a self-dual structure of positive type on $m \boldsymbol{P}^{2}, m>0$. In this section we shall show that the algebraic dimension $a(Z)$ of $Z$ equals two, provided that $Z$ contains a special rational surface as considered in Lemma 3.2 as a smooth member of the system $\left|K^{-1 / 2}\right|$, where $K:=K_{Z}$ is the canonical bundle of $Z$ and $K^{1 / 2}$ is its canonical square root.

Suppose now that $\left|K^{-1 / 2}\right|$ contains a smooth member $S$, which is necessarily a rational surface (cf. [PP94a]). By the Hitchin vanishing theorem $H^{2}\left(Z, O_{Z}\right)=0$ (see [Hit80]). Then we get $H^{1}\left(Z, O_{Z}\right)=0$ since $\chi\left(Z, O_{Z}\right)=c_{1} c_{2} / 24=1 / 2(\chi-\tau)=1$ (cf. [Hit81]), where $\chi$ and $\tau$ are respectively the Euler characteristic and the signature of $m \boldsymbol{P}$. Then in view of the relation $\left.K^{-1 / 2}\right|_{S}=K_{S}^{-1}$ we get the exact sequence of sheaves

$$
0 \rightarrow O_{Z} \rightarrow K^{-1 / 2} \rightarrow K_{S}^{-1} \rightarrow 0
$$

and the associated exact sequence of cohomology

$$
0 \rightarrow H^{0}\left(Z, O_{Z}\right) \rightarrow H^{0}\left(Z, K^{-1 / 2}\right) \stackrel{b}{\rightarrow} H^{0}\left(S, K_{S}^{-1}\right) \rightarrow 0
$$

In particular we have $h^{0}\left(K^{-1 / 2}\right)=1+h^{0}\left(K_{S}^{-1}\right)$. Suppose that $h^{0}\left(K_{S}^{-1}\right)>0$ so that $\operatorname{dim}\left|K^{-1 / 2}\right| \geqslant 1$. Let $P$ be a linear subpencil of $\left|K^{-1 / 2}\right|$ whose general member is smooth. Then there exists a countable or finite subset $N$ of $P$ such that for any $y \in P-N$ the corresponding surface $S_{y}$ on $Z$ is nonsingular and its anti-Kodaira dimension $\kappa^{-1}\left(S_{y}\right)=d$ for some fixed integer $d=d(P), 0 \leqslant d \leqslant 2$. We shall relate this number $d$ with the algebraic dimension $a(Z)$ of $Z$.

Proposition 4.1. Let $P$ be a linear pencil with $d=d(P)$ as above. Then we have the equality $a(Z)=d+1$. In particular $d=d(P)$ is independent of the choice of such a $P$.

Proof. Let $S$ be any general smooth member of $P$. Then we have the inequality

$$
a(Z) \leqslant \kappa^{-1}(S)+1=d+1
$$

by [Cam91, Proposition 1.5]. It thus suffices to show the reverse inequality $a(Z) \geqslant d+1$. By eliminating the indeterminacy of the pencil $P$ and taking a resolution of the singularities we obtain the diagram

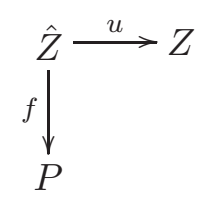

of compact complex manifolds, where $u$ is a bimeromorphic morphism; moreover, the proper transform $\hat{S}$ of a general member $S$ of $P$ in $\hat{Z}$ is a fiber of $f$ and is mapped isomorphically onto $S$ by $u$. Then by [Uen75, Proposition 12.2] we get that

$$
a(\hat{Z}) \geqslant d+\operatorname{dim} P=d+1=\kappa^{-1}(S)+1 .
$$

Suppose further that $S$ is isomorphic to a nonsingular rational surface $S_{a}$ for some $a=$ $\left(a_{1}, \ldots, a_{4}\right) \in U$ defined before Lemma 3.2. We identify $S$ with $S_{a}$ in what follows. Surface $S$ contains a cycle $C_{a}$ of nonsingular rational curves, which is a member of the anti-canonical system $\left|K_{S}^{-1}\right|$. 


\section{A. FujIKI}

If $\left|K^{-1 / 2}-C_{a}\right|$ denotes the linear subsystem of $\left|K^{-1 / 2}\right|$ consisting of elements which contain $C_{a}$, we have $\operatorname{dim}\left|K^{-1 / 2}-C_{a}\right|=1$ by (10).

Proposition 4.2. Suppose that the line bundle $L_{a}$ on $B$ in Lemma 3.2 is a torsion line bundle. Then we have $a(Z)=2$.

In view of Proposition 4.1, the proposition is a consequence of the following lemma.

Lemma 4.3. Let $P=\left|K^{-1 / 2}-C_{a}\right|$, which is a subpencil of $\left|K^{-1 / 2}\right|$ containing $S$. Then under the assumption of the previous proposition we have $d(P)=\kappa^{-1}(S)=1$.

Proof. Let a point $o \in P$ correspond to $S$. Then by the definition of $d(P)$ it suffices to show that there exists a neighborhood $U$ of $o$ in $P$ such that for any point $t \in U$ the corresponding surface $S_{t}$ is nonsingular and with $\kappa^{-1}\left(S_{t}\right)=1$.

For this purpose first we note that $C_{a}=C_{1}+\cdots+C_{2 k}, k=m+2$, is naturally contained in every $S_{t}$ and recall a sequence of bimeromorphic morphisms $w_{a}: S=S_{a} \rightarrow T^{\prime}, u: T^{\prime} \rightarrow T$ and $\alpha: T \rightarrow S_{0}$ of nonsingular surfaces, where $w_{a}$ contracts four $(-1)$-curves $D_{a i}, 1 \leqslant i \leqslant 4$, in $S_{a}$ to the smooth points $a_{i}^{\prime}$ of $B^{\prime} \subseteq T^{\prime}$ and $u$ and $\alpha$ are admissible bimeromorphic morphisms. More precisely, we have decompositions $u=\mu_{m-2} \cdots \mu_{1}$ and $\alpha=\mu_{m-1} \mu_{m}$, where $\mu_{i}: T_{i-1} \rightarrow T_{i}$ are admissible blowing-downs with $T_{0}=T^{\prime}$ and $T_{m}=S_{0}$, and for each $i$ there exists a unique index $\kappa_{i}, 1 \leqslant \kappa_{i} \leqslant k$ (with $\kappa_{i} \neq \kappa_{j}$ for $i \neq j$ ) such that if we set $\nu_{i}:=\mu_{i} \cdots \mu_{1}: T^{\prime} \rightarrow T_{i}, 1 \leqslant i \leqslant m$, and $\nu_{0}:=i d_{T^{\prime}}$, then $\nu_{i-1}\left(C_{\kappa_{i}}\right)$ and $\nu_{i-1}\left(C_{\kappa_{i}+k}\right)$ are $(-1)$-curves on $T_{i-1}$ and $\mu_{i}$ is precisely the contraction of these two $(-1)$-curves.

We also recall a general fact: Let $\left\{X_{t}\right\}_{t \in A}$ be a holomorphic family of nonsingular complex surfaces parametrized by a domain $A$ in $C$. Let $C$ be a (-1)-curve on $X_{o}$ for some $o \in A$. Then there exist a neighborhood $U$ of $o$ in $A$ and a unique holomorphic family $\left\{C_{t}\right\}$ of $(-1)$-curves $C_{t}$ in $X_{t}$ such that $C_{o}=C$ (cf. [Kod63]). Moreover, there exists a holomorphic family of bimeromorphic morphisms $X_{t} \rightarrow Y_{t}, t \in U$, which blow down $C_{t}$ to nonsingular points $p_{t}$ of $Y_{t}$ (cf. [FN72]).

Applying this result to the family $\left\{S_{t}\right\}_{t \in P}$ in a neighborhood of $o$ and the $(-1)$-curves $D_{a i}, 1 \leqslant$ $i \leqslant 4$, in $S$ we have the following: There exist a neighborhood $U$ of $o$ in $P$, holomorphic families of $(-1)$-curves $\left\{D_{a i, t}\right\}_{t \in U}$ in $S_{t}$ with $D_{a i, o}=D_{a i}, 1 \leqslant i \leqslant 4$, and a holomorphic family of bimeromorphic morphisms $w_{t}: S_{t} \rightarrow T_{t}^{\prime}$ contracting $D_{a i, t}$ to points where $T_{o}^{\prime}=T^{\prime}$ and $w_{o}=w_{a}$. Here, if we take $U$ sufficiently small, we can assume that each $D_{a i, t}$ intersects transversally at a single point with a unique irreducible component of $C_{a}$ which is independent of $t$. Thus $C_{a}$ is mapped isomorphically onto a curve in $T_{t}^{\prime}$ denoted again by $C_{a}$ independently of $t$.

The self-intersection number of each irreducible component of $C_{a}$ in $T_{t}^{\prime}$ is independent of $t$; in particular $C_{\kappa_{1}}$ and $C_{\kappa_{1}+k}$ are considered as $(-1)$-curves in $T_{t}^{\prime}$ for all $t$. Then $\mu_{1}$ extends to a holomorphic family of admissible blowing-downs $\mu_{1 t}: T_{0 t}:=T_{t}^{\prime} \rightarrow T_{1 t}$ which are indeed the blowing-downs of these (-1)-curves in $T_{t}^{\prime}$ with $\mu_{1 o}=\mu_{1}$ and $T_{1 o}=T_{1}$. Clearly the restriction $\left.\mu_{1 t}\right|_{C_{a}}$ is independent of $t$ and hence so is the image $C_{a t}^{(1)}:=\mu_{1 t}\left(C_{a}\right)$ in $T_{1 t}$ up to isomorphisms together with the self-intersection numbers of its irreducible components.

Then $\mu_{1 t}\left(C_{\kappa_{2}}\right)$ and $\mu_{1 t}\left(C_{\kappa_{1}+k}\right)$ are $(-1)$-curves in $T_{1 t}$ and we have again a holomorphic family of admissible blowing-downs $\mu_{2 t}: T_{1 t} \rightarrow T_{2 t}$ contracting the two curves with $\mu_{2 o}=\mu_{2}$ and $T_{2 o}=T_{2}$. Continuing in this way we finally obtain holomorphic families of admissible blowingdowns $\mu_{i t}: T_{i-1, t} \rightarrow T_{i t}$ with $\mu_{i o}=\mu_{i}$ and $T_{i o}=T_{i}, 1 \leqslant i \leqslant m$, such that the image $C_{a t}^{(i)}:=\nu_{i t}\left(C_{a}\right)$ is independent of $t$ up to canonical isomorphisms, where $\nu_{i t}=\mu_{i t} \cdots \mu_{1 t}$. In particular the pair $\left(T_{m t}, C_{a t}^{(m)}\right)$ is isomorphic to the pair $\left(T_{m o}, \nu_{m o}\left(C_{a}\right)\right)=\left(S_{0}, \nu_{m}\left(C_{a}\right)\right) \cong(\boldsymbol{P} \times \boldsymbol{P}, \bar{C})$ since the last pair is rigid under small (log-)deformations.

Then identifying $\left(T_{m t}, C_{a}^{(m)}\right)$ with $(\boldsymbol{P} \times \boldsymbol{P}, \bar{C})$ if we follow the above process of successive blowingdowns in the reverse way as a succession of admissible blowing-ups, we conclude that the blowing-up 


\section{TWistor SPACES OF ALGEBRAIC DIMENSION TWO}

occurs in each step on the same pairs of points independently of $t$, and in particular $\left(T_{t}^{\prime}, C_{a}\right)$ are all isomorphic to $\left(T^{\prime}, B\right)$ independently of $t$ (after possible restriction of $U$ ).

Thus taking and fixing isomorphisms of these spaces we shall suppress the suffix $t$; e.g. we write $T_{t}^{\prime}=T^{\prime}, T_{t}=T, S_{0 t}=S_{0}, u_{t}:=\mu_{m-2, t} \cdots \mu_{1 t}=u$, and $\alpha_{t}:=\mu_{m t} \mu_{m-1, t}=\alpha$. Accordingly, we consider $w_{t}$ as a bimeromorphic morphism $\left(S_{t}, C_{a}\right) \rightarrow\left(T^{\prime}, B^{\prime}\right)$, which is the blowing-down of four $(-1)$-curves $D_{i t}, 1 \leqslant i \leqslant 4$, in $S_{t}$ that are deformations of $D_{i o}=D_{a i}$. Thus if $a_{i t}^{\prime}=w_{t}\left(D_{i t}\right)$ are the points of $T^{\prime}$ which form the center of the blowing-up $w_{t}$, and $\left[D_{t}\right]$ is the line bundle on $S_{t}$ defined by the divisor $D_{t}:=D_{1 t}+D_{2 t}+D_{3 t}+D_{4 t}$, we get that $\left[D_{t}\right] \mid C_{a}$ is the pull-back of the line bundle $\tilde{F}_{a t}$ on $B^{\prime}$ defined by the Cartier divisor $a_{1 t}^{\prime}+a_{2 t}^{\prime}+a_{3 t}^{\prime}+a_{4 t}^{\prime}$ on $B^{\prime}$ with respect to the isomorphism $w_{t} \mid C_{a}: C_{a} \stackrel{\sim}{\rightarrow} B^{\prime}$, which is independent of $t$ and coincides with $w_{B}:=w \mid B$. Note that if we restrict $U$ smaller if necessary, $a_{i t}^{\prime}$ belongs to $E_{i}^{\prime}-\left\{q_{i}^{\prime}, r_{i}^{\prime}\right\}$.

Now since $K_{S_{t}}^{-1}\left|C_{a} \cong K^{-1 / 2}\right| C_{a}$ by the adjunction formula, $K_{S_{t}}^{-1} \mid C_{a}$ is independent of $t$. Furthermore we have

$$
\left(K_{S_{t}}^{-1} \otimes\left[D_{t}\right]\right)\left|C_{a} \cong\left(w_{t}^{*} K_{T^{\prime}}^{-1}\right)\right| C_{a}=\left(w_{t} \mid C_{a}\right)^{*}\left(K_{T^{\prime}}^{-1} \mid B^{\prime}\right)=w_{B}^{*}\left(K_{T^{\prime}}^{-1} \mid B^{\prime}\right) .
$$

Hence we get

$$
\left(w_{t} \mid C_{a}\right)^{*} \tilde{F}_{a t} \cong\left(K_{S_{t}} \mid C_{a}\right) \otimes w_{B}^{*}\left(K_{T^{\prime}}^{-1} \mid B^{\prime}\right) .
$$

By what we have shown above, the right-hand side is independent of $t$, and hence the same is true for the left-hand side. Moreover, since $\left(w_{t} \mid C_{a}\right)_{*} O_{C_{a}}^{*} \cong O_{B^{\prime}}^{*}, w_{t}^{*}: \operatorname{Pic} B^{\prime} \rightarrow \operatorname{Pic} C_{a}$ is injective. This implies that $\tilde{F}_{a t}$ itself is independent of $t$ as an element of Pic $B^{\prime}$. Furthermore, since $u \mid B^{\prime}$ : $B^{\prime} \rightarrow B$ induces isomorphisms of $E_{i}^{\prime}$ and $E_{i}$, we have $\tilde{F}_{a t} \cong u^{*} F_{a t}$, where $F_{a t}$ is the line bundle on $B$ defined by the Cartier divisor $a_{1 t}+a_{2 t}+a_{3 t}+a_{4 t}$ on $B$ with $a_{i t}=u\left(a_{i t}^{\prime}\right)$.

Thus if we set $a_{t}=\left(a_{1 t}, \ldots, a_{4 t}\right)$, we can identify $S_{t}$ with $S_{a_{t}}$ and $F_{a t}$ with $F_{a_{t}}$ in the notation of Lemma 3.2, and therefore $L_{a_{t}}:=F_{a_{t}}^{-1} \otimes\left(K_{T}^{-1} \mid B\right)$ is also independent of $t$ as a line bundle on $B$. In particular it is a torsion line bundle on $B$ as well as $L_{a}$. Hence, by Lemma $3.2, \kappa^{-1}\left(S_{t}\right)=$ $\kappa^{-1}\left(S_{a_{t}}\right)=1$ for all $t \in U$ as was desired.

\section{Deformations of Moishezon twistor spaces}

Let $Z$ be a Moishezon twistor space associated to a compact self-dual manifold $(M,[g])$ with $M=m \boldsymbol{P}^{2}, m>0$, which is necessarily of positive type [Poo88]. Suppose that $Z$ contains a smooth member $S$ of $\left|K^{-1 / 2}\right|$, which is a rational surface (cf. [PP94a]). In this section we show the vanishing of the obstructions related to deformations of $Z$ and $S$. Similar but somewhat weaker results can be found in the articles by Campana [Cam91, Cam94], LeBrun [LeB92], and Pedersen and Poon [PP94b]. The proof of the following theorem is given after Lemma 5.5

THEOREM 5.1.

1) For $i=2,3$ we have

$$
H^{i}(Z, \Theta)=H^{i}(Z, \Theta(-\log S))=H^{i}(Z, \Theta(-S))=H^{i}(S, \Theta)=0 .
$$

2) The natural map $H^{1}(Z, \Theta(-\log S)) \rightarrow H^{1}(S, \Theta)$ is surjective.

3) We have

$$
h^{1}(Z, \Theta)-h^{0}(Z, \Theta)=7 m-15, \quad h^{1}\left(\Theta_{Z}(-\log S)\right)-h^{0}\left(\Theta_{Z}(-\log S)\right)=5 m-6 .
$$

Here $\Theta$ denotes the sheaf of germs of holomorphic vector fields on $Z$, and $\Theta(-\log S)$ (respectively $\Theta(-S)$ ) the subsheaf of $\Theta$ defined by those vector fields which are tangent to (respectively vanish on) $S$. Note that if $H^{2}(Z, \Theta(-\log S))$ vanishes, then the local deformations of the pair $(Z, S)$ 


\section{A. FujikI}

are unobstructed and the tangent space of the corresponding Kuranishi space is naturally identified with $H^{1}(Z, \Theta(-\log S))$. In particular we obtain the following corollary.

Corollary 5.2. The Kuranishi spaces $T_{Z}, T_{S}$ and $T_{(Z, S)}$ for the local deformations of $Z, S$ and the pair $(Z, S)$ respectively are all smooth. The versal holomorphic maps $T_{(Z, S)} \rightarrow T_{S}$ are submersive at the base point.

Let $\Omega=\Omega_{Z}^{1}$ be the sheaf of holomorphic 1-forms on $Z$. Applying $\otimes K^{1 / 2}$ to (9) we obtain a short exact sequence

$$
0 \rightarrow K \rightarrow K^{1 / 2} \rightarrow K_{S} \rightarrow 0
$$

Applying $\otimes \Omega$ to $(9)$ and to this sequence we further get

$$
\left.0 \rightarrow \Omega K^{1 / 2} \rightarrow \Omega \rightarrow \Omega\right|_{S} \rightarrow 0
$$

and

$$
\left.0 \rightarrow \Omega K \rightarrow \Omega K^{1 / 2} \rightarrow \Omega\right|_{S} K_{S} \rightarrow 0,
$$

respectively. (Here and in what follows the juxtaposition denotes the tensor product.) Consider the associated long cohomology exact sequences

$$
\rightarrow H^{0}\left(S,\left.\Omega\right|_{S}\right) \rightarrow H^{1}\left(Z, \Omega K^{1 / 2}\right) \rightarrow H^{1}(Z, \Omega) \stackrel{a}{\rightarrow} H^{1}\left(S,\left.\Omega\right|_{S}\right) \rightarrow
$$

and

$$
\rightarrow H^{0}\left(S,\left.\Omega\right|_{S} K_{S}\right) \rightarrow H^{1}(Z, \Omega K) \rightarrow H^{1}\left(Z, \Omega K^{1 / 2}\right) \rightarrow H^{1}\left(S,\left.\Omega\right|_{S} K_{S}\right) \rightarrow .
$$

On the other hand, by applying $\otimes K_{S}$ to the standard exact sequence

$$
\left.0 \rightarrow N^{*} \rightarrow \Omega\right|_{S} \rightarrow \Omega_{S} \rightarrow 0,
$$

we obtain

$$
\left.0 \rightarrow N^{*} K_{S} \rightarrow \Omega\right|_{S} K_{S} \rightarrow \Omega_{S} K_{S} \rightarrow 0,
$$

where $N$ is the normal bundle of $S$ in $Z$ and $N^{*}$ its dual. From these we get the associated cohomology exact sequences

$$
\begin{aligned}
0 & \rightarrow H^{0}\left(S, N^{*}\right) \rightarrow H^{0}\left(S,\left.\Omega\right|_{S}\right) \rightarrow H^{0}\left(S, \Omega_{S}\right) \\
& \rightarrow H^{1}\left(S, N^{*}\right) \rightarrow H^{1}\left(S,\left.\Omega\right|_{S}\right) \stackrel{b}{\rightarrow} H^{1}\left(S, \Omega_{S}\right) \rightarrow
\end{aligned}
$$

and

$$
0 \rightarrow H^{0}\left(S, N^{*} K_{S}\right) \rightarrow H^{0}\left(S,\left.\Omega\right|_{S} K_{S}\right) \rightarrow H^{0}\left(S, \Omega_{S} K_{S}\right) \rightarrow H^{1}\left(S, N^{*} K_{S}\right) \rightarrow .
$$

Since $S$ is rational, in view of $N^{*}=K_{S}$ and $N^{*} K_{S}=2 K_{S}$ we see that $H^{0}\left(S, N^{*}\right)=H^{0}\left(S, N^{*} K_{S}\right)=0$, $H^{1}\left(S, N^{*}\right)=0$ and $H^{0}\left(S, \Omega_{S} K_{S}\right)=0$. Substituting these into (13) and (14) we get the next lemma.

Lemma 5.3. We have that $H^{0}\left(S,\left.\Omega\right|_{S}\right)=0$ and $H^{0}\left(S,\left.\Omega\right|_{S} K_{S}\right)=0$.

Next we prove the following.

Lemma 5.4. The restriction map $H^{1}(Z, \Omega) \rightarrow H^{1}\left(S, \Omega_{S}\right)$ is injective.

Proof. Since both $Z$ and $S$ are Moishezon with $h^{2,0}=0$, it suffices to show the injectivity of the restriction map $r: H^{2}(Z, C) \rightarrow H^{2}(S, C)$. Let $t: Z \rightarrow M$ be the $C^{\infty}$ twistor fibration. We consider the commutative diagram

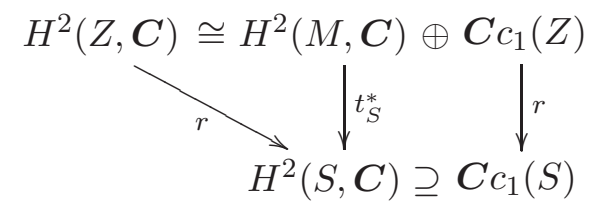




\section{TWistor SPACES OF ALGEBRAIC DIMENSION TWO}

where the horizontal isomorphism is via the Leray-Hirsch theorem (cf. [Hit81]), $t_{S}: S \rightarrow M$ is the restriction of $t$ to $S$ and $c_{1}$ denotes the first Chern class.

We know the injectivity of $t_{S}^{*}$; indeed, if we describe $S$ as a surface obtained from $\boldsymbol{P} \times \boldsymbol{P}$ by a succession of blowing-ups of pairwise $\sigma$-conjugate points, the Chern classes $c(i)$ of the exceptional curves $E_{i}+\bar{E}_{i}$ in $S$ coming from the $i$ th blowing-up forms a basis of $t_{S}^{*} H^{2}(M, C)$ (cf. [PP94a, $\left.\S 3\right]$ ). Moreover, $c_{1}(S)$ is not a linear combination of $c(i)$ in $H^{2}(S, \boldsymbol{C})$. Thus the injectivity follows from the above diagram.

Applying these to the above sequences (11) and (12) we obtain our next lemma.

Lemma 5.5. We have that $H^{i}(Z, \Omega K)=H^{i}\left(Z, \Omega K^{1 / 2}\right)=0, i=0,1$.

Proof. The assertion for $i=0$ follows from the fact that $K^{-1 / 2}$ is effective and $H^{0}(Z, \Omega)=0$ (cf. [Hit81]). For $i=1$ we note that the restriction map $H^{1}(Z, \Omega) \rightarrow H^{1}\left(S, \Omega_{S}\right)$ factors as

$$
H^{1}(Z, \Omega) \stackrel{a}{\rightarrow} H^{1}\left(S,\left.\Omega\right|_{S}\right) \stackrel{b}{\rightarrow} H^{1}\left(S, \Omega_{S}\right)
$$

Since $b a$ is injective by Lemma $5.4, a$ also is injective. Thus in view of (11) and Lemma 5.3 we get $H^{1}\left(Z, \Omega K^{1 / 2}\right)=0$, which in turn yields the vanishing of $H^{1}(Z, \Omega K)$ in view of (12) and Lemma 5.3.

Proof of Theorem 5.1. By Serre duality and Lemma 5.5 we get

$$
H^{i}\left(Z, \Theta_{Z}\right)=H^{i}\left(Z, \Theta_{Z}(-S)\right)=0 \text { for } i=2,3 .
$$

Consider the long exact sequence

$$
\begin{aligned}
0 & \rightarrow H^{0}(Z, \Omega K(\log S)) \rightarrow H^{0}\left(Z, \Omega K^{1 / 2}\right) \rightarrow H^{0}\left(S, \Omega_{S} K_{S}\right) \rightarrow \\
& \rightarrow H^{1}(Z, \Omega K(\log S)) \rightarrow H^{1}\left(Z, \Omega K^{1 / 2}\right) \rightarrow H^{1}\left(S, \Omega_{S} K_{S}\right) \rightarrow
\end{aligned}
$$

associated to the sheaf exact sequence

$$
0 \rightarrow \Omega K(\log S) \rightarrow \Omega(S) K \rightarrow \Omega_{S} K_{S} \rightarrow 0,
$$

where we have used the isomorphism $\Omega(S) K \cong \Omega\left(K^{1 / 2}\right)$. By Lemma $5.5, H^{i}\left(Z, \Omega K^{1 / 2}\right)$ vanish for $i=0,1$ and $H^{0}\left(S, \Omega_{S} K_{S}\right)=0$ since $S$ is rational. Thus we get

$$
H^{0}(Z, \Omega K(\log S))=H^{1}(Z, \Omega K(\log S))=0 .
$$

By taking the Serre dual we get

$$
H^{2}(Z, \Theta(-\log S))=H^{3}(Z, \Theta(-\log S))=0 .
$$

Thus part 1 is proved. Further, from the exact sequence of cohomology

$$
0 \rightarrow H^{0}\left(S, \Theta_{S}\right) \rightarrow H^{1}(Z, \Theta(-S)) \rightarrow H^{1}(Z, \Theta(-\log S)) \rightarrow H^{1}\left(S, \Theta_{S}\right) \rightarrow 0,
$$

arising from the sheaf exact sequence

$$
0 \rightarrow \Theta(-S) \rightarrow \Theta(-\log S) \rightarrow \Theta_{S} \rightarrow 0,
$$

we get part 2 of the theorem.

In view of the Riemann-Roch theorem and the relations (cf. [Hit81])

$$
c_{1}^{3}=16(2 \chi-3 \tau), \quad c_{1} c_{2}=12(\chi-\tau) \quad \text { and } \quad c_{3}=2 \chi,
$$

where $c_{i}$ are Chern classes of $Z$, and $\chi$ and $\tau$ are the Euler number and the signature of $M$ respectively, we obtain

$$
\chi(Z, \Theta)=\frac{1}{2}\left\{4\left(12 c_{1}^{3}-19 c_{1} c_{2}+12 c_{3}\right)\right\}=\frac{15}{2} \chi-\frac{19}{2} \tau=-7 m+15,
$$




\section{A. FujIKI}

since $\chi=m+2$ and $\tau=m$. Hence by part 1 we have

$$
h^{1}(Z, \Theta)-h^{0}(Z, \Theta)=7 m-15 .
$$

Further, from the sheaf exact sequence

$$
0 \rightarrow \Theta_{Z}(-\log S) \rightarrow \Theta_{Z} \rightarrow N_{S / Z} \rightarrow 0
$$

we get

$$
\chi\left(\Theta_{Z}(-\log S)\right)=\chi\left(\Theta_{Z}\right)-\chi\left(S, K_{S}^{-1}\right)=-7 m+15-\left(c_{1}^{2}(S)+1\right)=-5 m+6 .
$$

Hence by part 1 we have

$$
h^{1}\left(\Theta_{Z}(-\log S)\right)-h^{0}\left(\Theta_{Z}(-\log S)\right)=5 m-6 .
$$

Thus part 3 is proved. This completes the proof of Theorem 5.1.

Let $K:=S^{1} \times S^{1}$ be the real two-torus and $G:=C^{*} \times C^{*}$ its complexification. Let $m$ be a positive integer and fix a smooth effective action of $K$ on $m \boldsymbol{P}^{2}$. In [Joy95] Joyce has constructed a family of $K$-invariant self-dual metrics on $m \boldsymbol{P}^{2}$. We call a twistor space corresponding to any such invariant self-dual metric a Joyce twistor space (associated to the given $K$-action on $m \boldsymbol{P}^{2}$ ). It admits a naturally induced biholomorphic $G$-action. The structure of a Joyce twistor space has been studied in detail in [Fuj00]. In particular it is Moishezon.

The class of Joyce twistor spaces contains as a special case the degenerate case of LeBrun's twistor spaces [LeB91], which we call of LeBrun type (cf. [Fuj00, Proposition 6.14] for the details). Now we specialize the above consideration to the case of Joyce twistor spaces and determine the dimensions of the individual cohomology groups in Theorem 5.1.

Proposition 5.6. In Theorem 5.1 suppose that $Z$ is a Joyce twistor space and $S$ is $G$-invariant (which is automatic unless $Z$ is of LeBrun type). Suppose further that $m \geqslant 3$. Then we have

1) $h^{0}\left(\Theta_{Z}(-\log S)\right)=2$ and $h^{1}\left(\Theta_{Z}(-\log S)\right)=5 m-4$

2) $h^{0}\left(Z, \Theta_{Z}\right)=2$ and $h^{1}(Z, \Theta)=7 m-13$.

Proof. The assertion on $h^{1}$ follows from the assertion on $h^{0}$ by Theorem 5.1. So we shall compute $h^{0}$. (For the details on the structure of $Z$ used below we refer to [Fuj00, §6].) Since $Z$ admits a $G$-action which preserves $S$, it is clear that $2 \leqslant h^{0}\left(\Theta_{Z}(-\log S)\right) \leqslant h^{0}\left(\Theta_{Z}\right)$. Thus it suffices to show that $h^{0}\left(\Theta_{Z}\right)=2$.

First we show this assuming that $Z$ is not of LeBrun type. (In particular $m \geqslant 3$.) In this case $\left|K^{-1 / 2}\right|$ is a pencil and its base locus $C$ is a cycle of nonsingular rational curves. Let $\mu: \hat{Z} \rightarrow Z$ be the blowing-up of $Z$ with center $C$. Then $\hat{Z}$ becomes a holomorphic fiber space $f: \hat{Z} \rightarrow P$ over a nonsingular rational curve $P$ and $E:=\mu^{-1}(C)$ is isomorphic to the product $C \times P$. Clearly, the biholomorphic automorphism group Aut $Z$ of $Z$ preserves $\left|K^{-1 / 2}\right|$ and hence the action lifts to one on $\hat{Z}$ preserving the fibers of $f$ and $E$. Note that the induced action of $A_{u t} Z$ on $\left|K^{-1 / 2}\right|$ is trivial since for $m \geqslant 3$ it contains more than three singular fibers as pointed out below. Let $\operatorname{Aut}(\hat{Z}, E) / P$ be the relative Lie group over $P$ whose fiber over a point $y \in P$ is just the automorphism group of the pair $\left(Z_{y}, E_{y}\right)$. Then an irreducible component of $\operatorname{Aut}(\hat{Z}, E) / P$ which contains the identity section and is mapped surjectively onto $P$ is unique and is isomorphic to $G \times P$. Since only holomorphic sections of the projection $G \times P \rightarrow P$ are constant sections, the identity component $\mathrm{Aut}_{0} Z$ must coincide with $G$. It follows that $h^{0}\left(Z, \Theta_{Z}\right)=\operatorname{dim} G=2$. Thus the non-LeBrun case is finished.

In the LeBrun case the linear subsystem $\left|K^{-1 / 2}\right|^{G}:=\boldsymbol{P}\left(H^{0}\left(Z, K^{-1 / 2}\right)^{G}\right)$ of $\left|K^{-1 / 2}\right|$ is a pencil. There exist $m+2$ pairs $\left(S_{i}^{+}, S_{i}^{-}\right), 1 \leqslant i \leqslant m+2$, of $\sigma$-conjugate divisors such that $S_{i}:=S_{i}^{+}+S_{i}^{-}$ belongs to $\left|K^{-1 / 2}\right|^{G}$. Moreover, one can check that $S_{i}^{ \pm}$do not move in $Z$ except for exactly two successive $i$, say $m+1$ and $m+2$, if $m \geqslant 3$ (cf. [Fuj00, Lemmas 6.13 and 2.10]). In this case 


\section{TWistor SPACES OF ALGEBRAIC DIMENSION TWO}

$S_{i}^{ \pm}, i \neq m+1, m+2$, are preserved by the action of $\mathrm{Aut}_{0} Z$. Since $S_{1}$ and $S_{2}$ generate the pencil $\left|K^{-1 / 2}\right|^{G}$, $\operatorname{Aut}_{0} Z$ also preserves $\left|K^{-1 / 2}\right|^{G}$. The rest of the argument is then precisely the same as in the non-LeBrun case, starting from $\left|K^{-1 / 2}\right|^{G}$ instead of $\left|K^{-1 / 2}\right|$.

Remark. For general LeBrun twistor spaces we have $h^{1}\left(\Theta_{Z}(-\log S)\right)=5 m-5$ [PP94b, Theorem 2.3] and $h^{1}(Z, \Theta)=7 m-14$ [LeB92, p. 302, Corollary 1] for $m \geqslant 3$.

\section{Proof of Main Theorem}

Recall that a member of the system $\left|K^{-1 / 2}\right|$ is called a fundamental divisor. Let $S=S_{a \#}$ be the symmetric toric surface defined before Proposition 3.5. It has the natural real structure induced from that of $S_{0}$ (cf. $\S 2.2$ ). Then the second Betti number $b_{2}$ of $S$ is always even and $\geqslant 10$ by our construction. We start with the following proposition.

Proposition 6.1. There exists a Joyce twistor space $Z$ associated to a self-dual structure on $m \boldsymbol{P}^{2}$ with $2 m+2=b_{2}$ such that it contains the surface $S$ as a real smooth fundamental divisor, where the real structure on $S$ induced from $Z$ coincides with the original one. In particular $m \geqslant 4$.

Proof. Set $k=b_{2} / 2+1$. By our construction of $S$ we have $b_{2} \geqslant 10$ and hence $k \geqslant 6$. The toric surface $S$ is determined by the associated fan which consists of primitive elements $\rho_{i}=\left(s_{i}, t_{i}\right)$ in $Z^{2}, 1 \leqslant i \leqslant 2 k$, arranged counterclockwise such that $\left(s_{i+k}, t_{i+k}\right)=-\left(s_{i}, t_{i}\right)$ for $1 \leqslant i \leqslant k$, where $s_{i}$ and $t_{i}$ are coprime integers. We then define $\left(m_{i}, n_{i}\right):=\left(-t_{i}, s_{i}\right)$ for $1 \leqslant i \leqslant k$. The smoothness of $S$ implies for the determinant that

$$
\left|\begin{array}{cc}
m_{i} & n_{i} \\
m_{i+1} & n_{i+1}
\end{array}\right|=\left|\begin{array}{cc}
s_{i} & t_{i} \\
s_{i+1} & t_{i+1}
\end{array}\right|=1 .
$$

Hence the sequence $\left\{ \pm\left(m_{i}, n_{i}\right)\right\}_{1 \leqslant i \leqslant k}$ is the Orlik-Raymond invariant of a unique smooth $K$-action on $m \boldsymbol{P}^{2}$, where $m=k-2 \geqslant 4$ (cf. [Fuj00, (3.3) and (3.4)] and [Joy95, 3.1]). Take any of the Joyce twistor spaces $Z$ associated to this action. Then by [Fuj00, Lemma 5.12] together with the results of [Fuj00, §6] $Z$ contains a real smooth $G$-invariant fundamental divisor, which is isomorphic to the original $S$ and will be identified with $S$. The final assertion on the real structure on $S$ then follows from the general result of Pedersen and Poon [PP94a, §3] on the induced real structure of a real fundamental divisor.

Remark. If we take $S_{a}$ in such a way that each successive blowing-up forming the morphism $T^{\prime} \rightarrow T$ takes place always on the proper transform of $E_{1}$ and $E_{3}$, then the resulting twistor space is of LeBrun type and vice versa.

For the proof of the Main Theorem we need some remarks about the real structure on the spaces considered in $\S 5$. Let $Z$ be a Joyce twistor space. Then the linear system $\left|K^{-1 / 2}\right|$ contains the real pencil $\left|K^{-1 / 2}\right|^{G}$ whose general member $S$ is a smooth projective toric surface with respect to the induced $G$-action. Consider a triple $(Z, S,\{p, \bar{p}\})$ consisting of such a pair $(Z, S)$ with $S$ real and a pair $(p, \bar{p})$ of a point $p$ and its conjugate $\bar{p}:=\sigma(p)$, where $p$, and hence $\bar{p}$ also, is assumed to be in the open orbit $U$ on $S$. We then consider the local deformations $\left\{\left(Z_{t}, S_{t},\left\{p_{t}, q_{t}\right\}\right)\right\}_{t \in T}$ of the triple $(Z, S,\{p, \bar{p}\})$, where $Z_{t}$ is a compact complex threefold, $S_{t}$ a smooth divisor on $Z_{t}$, and $p_{t}$ and $q_{t}$ are two points of $S_{t}$. From Theorem 5.1, Corollary 5.2 and Proposition 5.6 we deduce easily that the Kuranishi family for such deformations of triples exists and its parameter space $T$ is smooth of dimension $5 m-2$. Moreover, $T$ admits a natural submersion $u$ onto the smooth parameter space of the Kuranishi family of the deformation of the pairs $(S,\{p, \bar{p}\})$ defined similarly.

On the other hand, since $p$ and $\bar{p}$ belong to $U$, by part 1 of Proposition 5.6 , the identity component of the automorphism group of the triple $(Z, S,\{p, \bar{p}\})$ (respectively the pair $(S,\{p, \bar{p}\})$ ) reduces to 


\section{A. FuJiki}

the identity. It follows that the above Kuranishi families are both universal. Therefore as in [DF89, Lemma 2.1] these Kuranishi families admit natural real structures and $u$ is compatible with them. In particular $u$ induces a submersion between the real parts of the parameter spaces.

Proof of the Main Theorem. Fix an integer $m \geqslant 4$. Consider the toric surface $S$ with real structure $\sigma$ in Proposition 6.1. By that proposition we can find a Moishezon twistor space $Z$ associated to a self-dual structure on $m \boldsymbol{P}^{2}$ which contains $S$ as a real fundamental divisor. Taking $n=1$ or 2 in Proposition 3.5 we find arbitrarily small deformations $\left(S_{a}, \sigma_{a}\right)$ of $(S, \sigma)$ as a surface with real structure such that $\kappa^{-1}\left(S_{a}\right)=1$. For any point $p$ of the open orbit $U$ of $S$ we can find real deformations $\left(S_{a},\left\{p_{a}, \bar{p}_{a}\right\}\right)$ of $(S,\{p, \bar{p}\})$ for a suitable choice of points $p_{a}$ of $S_{a}$, where $\bar{p}_{a}=\sigma_{a}\left(p_{a}\right)$. By the description preceding the proof these deformations of the pairs can be extended to real deformations $\left(Z_{a}, S_{a},\left\{p_{a}, \bar{p}_{a}\right\}\right)$ of the triple $(Z, S,\{p, \bar{p}\})$.

It is then known by a standard argument that $Z$ is a twistor space associated to some selfdual structure on $m \boldsymbol{P}^{2}$. Moreover, it contains $S_{a}$ as a fundamental divisor as follows the relations $l_{a} \cdot S_{a}=l \cdot S=2$ and $H^{1}\left(Z_{a}, O_{Z_{a}}\right)=0$, where $l_{a}$ and $l$ are twistor lines on $Z_{a}$ and $Z$ respectively. By Proposition 3.5 the line bundle $L_{a}$ in (6) is a torsion line bundle of order $n$. Thus, by Proposition $4.1, a\left(Z_{a}\right)=2$, in which case the corresponding self-dual metric is necessarily of positive type by [Pon91]. This completes the proof of the Main Theorem.

We conclude by making a remark on the case $m=4$. In this case the surface $S_{a}$ is an elliptic surface over $\boldsymbol{P}$ with a unique multiple fiber $C_{a}$ with multiplicity $n$, where $C_{a}$ is a cycle of eight nonsingular rational curves. Suppose that $S_{a}$ is realized as a real fundamental divisor of a twistor space of algebraic dimension two. Then we have seen that $n=1$ or 2 and in fact that both cases are actually realized. On the other hand, in the original examples of Campana and Kreußler [CK99] the corresponding fundamental divisor is an elliptic surface whose multiple fiber is a multiple of a nonsingular elliptic curve. Moreover, it is shown by Honda [Hon00] that the multiplicity $n$ can take arbitrary positive values.

\section{REFERENCES}

Cam91 F. Campana, The class $\mathcal{C}$ is not stable by small deformations, Math. Ann. 290 (1991), 19-30.

Cam94 F. Campana, The class $\mathcal{C}$ is not stable by small deformations II, Contemp. Math. 192 (1994), 65-76.

CK99 F. Campana and B. Kreußler, Existence of twistor spaces of algebraic dimension two over the connected sum of four complex projective planes, Proc. Amer. Math. Soc. 127 (1999), 2633-2642.

Dol81 I. Dolgacev, Algebraic surfaces with $p=p_{g}=0$, in Algebraic surfaces (Centro Internaz. Mat. Estivo, III Ciclo, Varenna-Como, 1977) (Liguori Editore, Napoli, 1981), 97-216.

DF89 S. Donaldson and R. Friedman, Connected sums of self-dual manifolds and deformations of singular spaces, Nonlinearity 2 (1989), 197-239.

Fuj00 A. Fujiki, Compact self-dual manifolds with torus actions, J. Differential Geom. 55 (2000), 229-324.

Fuj02 A. Fujiki, Topology of compact self-dual manifolds whose twistor space is of positive algebraic dimension, J. Math. Soc. Japan 54 (2002), 587-608.

FN72 A. Fujiki and S. Nakano, Supplement to 'On the inverse of monoidal transformations', Publ. Res. Inst. Math. Sci., Kyoto Univ. 7 (1972), 637-644.

Hit80 N. J. Hitchin, Linear field equations on self-dual space, Proc. Roy. Soc. London, Ser. A 370 (1980), 173-191.

Hit81 N. J. Hitchin, Kählerian twistor spaces, Proc. London Math. Soc. (3) 43 (1981), 133-150.

Hon99 N. Honda, Donaldson-Friedman construction and deformations of a triple of compact complex spaces, Osaka J. Math. 36 (1999), 641-672.

Hon00 N. Honda, On some twistor spaces over $4 \boldsymbol{C P}^{2}$, Compositio Math. 122 (2000), 323-336. 


\section{TWistor SPACES OF ALGEBRAIC DIMENSION TWO}

Joy95 D. Joyce, Explicit construction of self-dual 4-manifolds, Duke Math. J. 77 (1995), 519-552.

Kod63 K. Kodaira, On stability of compact submanifolds of complex manifolds, Amer. J. Math. 85 (1963), $79-94$.

LeB91 C. LeBrun, Explicit self-dual metrics on $\boldsymbol{C P}^{2} \# \cdot \cdots \# \boldsymbol{C} \boldsymbol{P}^{2}$, J. Differential Geom. 34 (1991), 223-253.

LeB92 C. LeBrun, Twistor, Kähler manifolds and bimeromorphic geometry I, J. Amer. Math. Soc. 5 (1992), 289-316.

LP92 C. LeBrun and Y. S. Poon, Twistors, Kähler manifolds, and bimeromorphic geometry II, J. Amer. Math. Soc. 5 (1992), 317-325.

PP94a H. Pedersen and Y. S. Poon, Self-duality and differentiable structures on the connected sum of complex projective planes, Proc. Amer. Math. Soc. 121 (1994), 859-864.

PP94b H. Pedersen and Y. S. Poon, A relative deformation of Moishezon twistor spaces, J. Algebraic Geom. 3 (1994), 685-701.

Pon91 M. Pontecorvo, Algebraic dimension of twistor spaces and scalar curvature of antiself dual metrics, Math. Ann. 291 (1991), 113-122.

Poo88 Y. S. Poon, Algebraic dimension of twistor spaces, Math. Ann. 282 (1988), 621-627.

Poo92 Y. S. Poon, On the algebraic structure of twistor spaces, J. Differential Geom. 36 (1992), 451-491.

Uen75 K. Ueno, Classification theory of compact complex manifolds, Lecture Notes in Mathematics, vol. 439 (Springer, Berlin, 1975).

Akira Fujiki fujiki@math.sci.osaka-u.ac.jp

Department of Mathematics, Graduate School of Science, Osaka University, Toyonaka, Osaka 560-0043, Japan 